\title{
The application of a long period grating sensors to human respiratory plethysmography
}

T. Allsop, K. Carroll, D. J. Webb, I. Bennion, Martin Miller

T. Allsop, K. Carroll, D. J. Webb, I. Bennion, Martin Miller, "The application of a long period grating sensors to human respiratory plethysmography," Proc. SPIE 6631, Novel Optical Instrumentation for Biomedical Applications III, 66311G (16 July 2007); doi: 10.1117/12.727821

SPIE. Event: European Conferences on Biomedical Optics, 2007, Munich, Germany 


\title{
The Application of a Long Period Grating Sensors to Human Respiratory Plethysmography
}

\author{
T. Allsop , K. Carroll, D.J. Webb, I. Bennion \\ Photonics Research Group, Aston University, Aston Triangle, Birmingham, B4 7ET, U.K. \\ t.d.p.allsop@aston.ac.uk
}

\author{
Martin. Miller \\ Respiratory Medicine, University Hospital Birmingham NHS Trust, University of Birmingham, \\ Birmingham, UK
}

\begin{abstract}
A series of nine in-line curvature sensors on a garment are used to monitor the thoracic and abdominal movements of a human during respiration for application to Human Respiratory Plethysmography. These results are used to obtain volumetric tidal changes of the human torso which show agreement with data from a spirometer used simultaneously to recorded the inspired and expired volume at the mouth during both rhythmic and transient breathing patterns. The curvature sensors are based upon long period gratings which are written in a progressive three layered fibre to render them insensitive to refractive index changes. The sensor consists of the long period grating laid upon a carbon fibre ribbon, with this then encapsulated in a low temperature curing silicone rubber. The sensing array is multiplexed and interrogated using a derivative spectroscopy based technique to monitor the response of the LPGs' attenuation bands to curvature. The versatility of this scheme is demonstrated by applying the same garment and sensors to various human body types and sizes. It was also found from statistical analysis of the sensing array data, in conjunction with the measurements taken with a spirometer, that 11 to 12 sensors should be required to obtain an absolute volumetric error of $5 \%$.
\end{abstract}

Keywords: Curvature Sensing, Long Period Gratings, Respiratory monitoring,

\section{INTRODUCTION}

Non-invasive measurement of thoracoabdominal surface motion can provide a comprehensive appreciation of respiratory function and enhance our understanding of respiratory physiology. Knowledge of the total and regional deflection of the surface during breathing not only provides a key to the volumetric flow of gases but also gives us an insight into the thoracic and abdominal contributions and the recruitment of muscles. Until now research in this field has been predominantly laboratory based and not portable which makes the available technology unsuitable for ambulatory or field applications. Konno and Mead [1] first demonstrated that respiratory gas flow could be measured at the surface of the chest and abdomen thus negating the need for measurement of flow at the mouth, which is the clinical standard at present. A number of measurement devices have been developed following Konno and Mead; paradigms include: Respiratory Inductive Plethysmography (RIP) [2], Optical Reflectance Plethysmography (ORP) [3] and the use of magnetometers [4]. Each of these has certain limitations for use in ambulatory respiration monitoring. These pertain to the accuracy over wide variations in tidal volume and changes in posture after the calibration of RIP [5]. This can be

Novel Optical Instrumentation for Biomedical Applications III, edited by Christian D. Depeursinge Proc. of SPIE-OSA Biomedical Optics, SPIE Vol. 6631, 66311G, @ 2007 SPIE-OSA · 1605-7422/07/\$18 
addressed by using a sensor array [6], however the solution relies heavily on model based signal processing. The cost and realisation of a sufficiently dense array of magnetometers is prohibitive. Whilst the ORP methods lack such limitations they rely on an off body reference with which to track specified anatomical locations. Present instrumentation is bulky and generally has to be operated while the patient occupies a confined area. It follows that the technique is not suitable for routine ambulatory monitoring. The fundamental requirement is to create a highly compliant sensor with which to track selected anatomical positions on the chest and abdomen surface. A possible solution to this problem is to use a fibre Long Period Grating (LPG) as the sensing element. Recent work [8,9] using fibre LPGs to monitor shape changes (bending) is promising but there is one major disadvantage: to interrogate LPGs, expensive broadband light sources and optical spectrum analysers (OSAs) are usually required.

This paper describes research investigating a possible solution to this problem involving an array of LPG curvature sensors interrogated by a set of distributed feedback lasers. Firstly, it is shown that this curvature sensor is suitable for the application of respiratory plethysmography. Secondly, the curvature sensors are adhered to a garment to enable them to be used to detect the shape variations of the chest and abdominal regions of a human torso during respiration using the transmission spectrum of the LPGs' attenuation bands. Thirdly, a curvature sensor array is used in conjunction with a signal-processing scheme based upon derivative spectroscopy [9] to show the multiplexing capability of the interrogation scheme and to demonstrate the monitoring of a human subject during respiration. Fourthly, analysis is performed on the acquired data to show that data from only six fully functional sensors is required to obtain approximately $6 \%$ volumetric error for the human subject used. Finally, the curvature sensing garment is used to monitor a variety of human subjects with body mass indices from 18 to 32; to the best of the authors' knowledge, this is the first application of a multiplexed LPG array. Potentially this sensing scheme can be both relatively inexpensive to implement and compact in size, meaning that the entire system could be attached to the patient being monitored.

\section{CURVATURE SENSOR}

The curvature sensor utilises an LPG as the sensing element. LPGs are photoinduced fibre devices that couple light from the core of a single-mode optical fibre into the cladding at discrete wavelengths and thereby generate attenuation bands in the transmission spectrum of the optical fibre core. Over the last few years the fibre LPG has found numerous applications in the field of sensing. This is because the LPGs are sensitive to strain $(\varepsilon)$, temperature $(T)$ and the surrounding refractive index $\left(n_{s}\right)[8,10]$. Most relevant to the current work, LPGs have also been used as curvature sensors $[11,12]$, since the LPG's attenuation bands are spectrally sensitive to bending, which induces both a wavelength shift and a change in the spectral profile of the attenuation band. The wavelength shift of the attenuation band arises from the phase matching condition of the LPG, given by [7]

$$
\lambda_{i}=\delta n_{\text {eff }} \Lambda \equiv\left[n_{\text {eff }}\left(\lambda_{l}, n_{1}, n_{2}, T, \mu \varepsilon, \boldsymbol{R}\right)-n^{v_{\text {eff }}} \text { cladding }_{i}\left(\lambda_{i}, n_{1}, n_{2}, T, \mu \varepsilon, n_{s}, \boldsymbol{R}\right)\right] \Lambda
$$

where $n_{\text {eff }}$ core is the effective index of the core mode and $n^{v}$ eff $_{\text {cladding }}$ is the effective index of the $v^{\text {th }}$ radial cladding mode, both indices being also dependent on the core and cladding refractive indices and wavelength $\lambda . \Lambda$ is the period of the grating. Whilst expression 1 gives the spectral position of the attenuation band, the magnitude of the spectral shift induced by the measurands $\left(\varepsilon, T, n_{s}\right)$ is dependent upon two factors: the difference between the effective refractive indices of the core and cladding modes and also the difference between the group effective refractive indices of these two modes [12]. The spectral sensitivity of LPGs to bending arises from two major components: the varying strain across the fibre and the effective change in the refractive index profile of the fibre induced by the bend itself. These can be visualised by the conformal mapping technique [13]. The bending of the optical fibre induces changes to the propagation constants of the cladding modes thus changing the group refractive indices as well as the effective refractive indices.

The main component of the curvature sensor is the LPG with a length of about $7 \mathrm{~cm}$ that is written in a progressive three layered fibre using the point by point method [14]. The result of using such a fibre is that the LPG produces attenuation bands that are insensitive to changes in the surrounding medium's refractive index. The LPG is laid upon a carbon fibre ribbon, this arrangement then being encapsulated in a low temperature $\left(90^{\circ} \mathrm{C}\right)$ curing silicone rubber - a Poly Siloxane 
polymer (Methyl Vinyl Silicone rubber). This type of silicone rubber is used to prevent significant changes to the LPG's attenuation bands due to thermal annealing. The use of the progressive three layered fibre prevents there from being any shift in the LPG's attenuation bands when it is embedded in the rubber. The sensor construct was designed to prevent the fibre from experiencing significant axial strain whilst at the same time providing a flexible stage for bending as well as a thermally insulating layer, reducing the effects of rapid ambient temperature fluctuations, see figure 1.

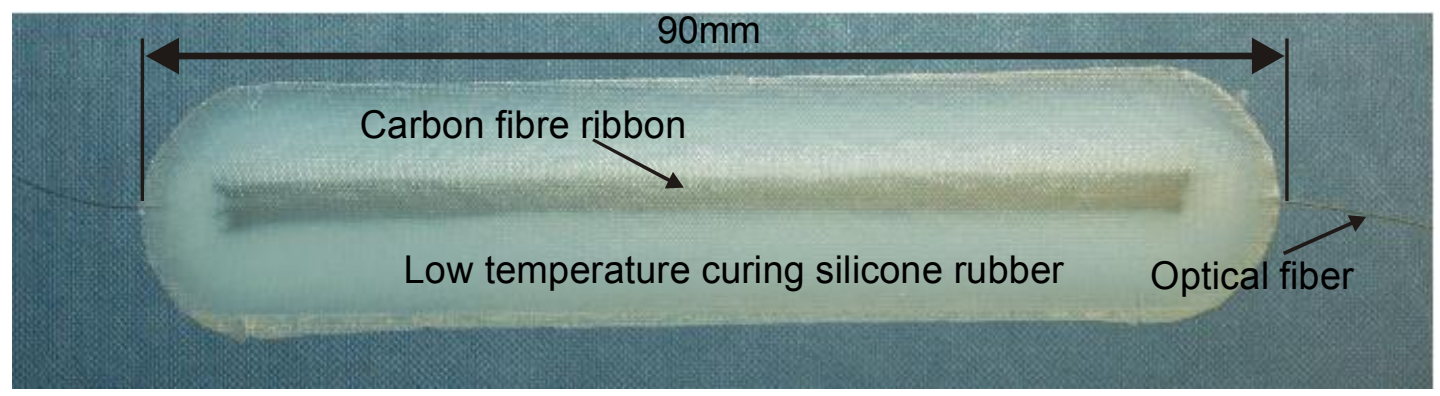

Figure 1. The curvature sensor

Measurements where preformed on the attenuation band associated with $\mathrm{HE}_{1,3}$ cladding mode at a wavelength of $1510 \mathrm{~nm}$ The temperature sensitivity was measured by immersing the sensor in a bath of hot water and monitoring the wavelength shift as the water cooled. This approach gave a sensitivity of $\mathrm{d} \lambda / \mathrm{dT}=0.0964 \pm 5 \times 10^{-4} \mathrm{~nm}^{\circ} \mathrm{C}^{-1}$. It should be noted, however, that this way of determining the temperature sensitivity may not necessarily correspond all that well to the way in which the sensor will be used in practice. The sensing fibre is embedded inside an insulating material and in the intended application, whilst one side of this material is exposed to the vagaries of the environment, the other side will be closer to body temperature, reducing the temperature variation experienced by the grating. It is worth noting that the temperature sensitivity of long period gratings is dependent upon the difference in thermo-optic coefficients of the materials used for core and cladding glass [9]; these can in principle be controlled to make the LPGs insensitive to temperature variations.

\section{INTERROGATION SCHEME}

The interrogation/multiplexing scheme implemented with these LPG curvature sensors is based upon derivative spectroscopy which was originally developed for wavelength modulation absorption spectrometry and usually involves applying a small sinusoidal wavelength modulation to the optical source wavelength [15]. This technique is mostly used for environmental monitoring and remote sensing as well as spectrochemical applications, e.g. $\mathrm{NO}_{2}$ and $\mathrm{CCL}_{4}$ detection.

We have implemented this approach to detect the changes in the spectral transmission profile of a LPG's stop band induced by the bending of the LPG. Modulating the DFB laser wavelength at a given frequency will generate a series of harmonics at the modulating frequency [16] in the output signal transmitted by the LPG. It has been shown that the in-phase component of the $\mathrm{n}^{\text {th }}$ harmonic output is proportional to the $\mathrm{n}^{\text {th }}$ derivative of the spectral profile under investigation [15]. The amplitudes of the first and second harmonics are therefore proportional to the first and second derivatives of spectral transmission function of the sensor, and the utility of our approach relies on the ratio of those derivatives being a unique function of the position in the spectral profile as well as being independent of any attenuation in the system. We have previously reported this interrogation technique in a system where a single LPG was interrogated using a pigtailed DFB laser as the light source [16].

The interrogation system used for the work reported in this paper employed a digital variant of this approach in which the laser's wavelength was cycled through three discrete values, with the transmitted intensity being recorded each time. The derivatives of the spectral profile were then approximated using these difference values, see Figure 2 . This 
digital derivative spectroscopy approach was more convenient to interface to the microcontroller based signal processing system developed for this project. In the final system two DFB lasers were used to address a total of up to ten curvature sensors

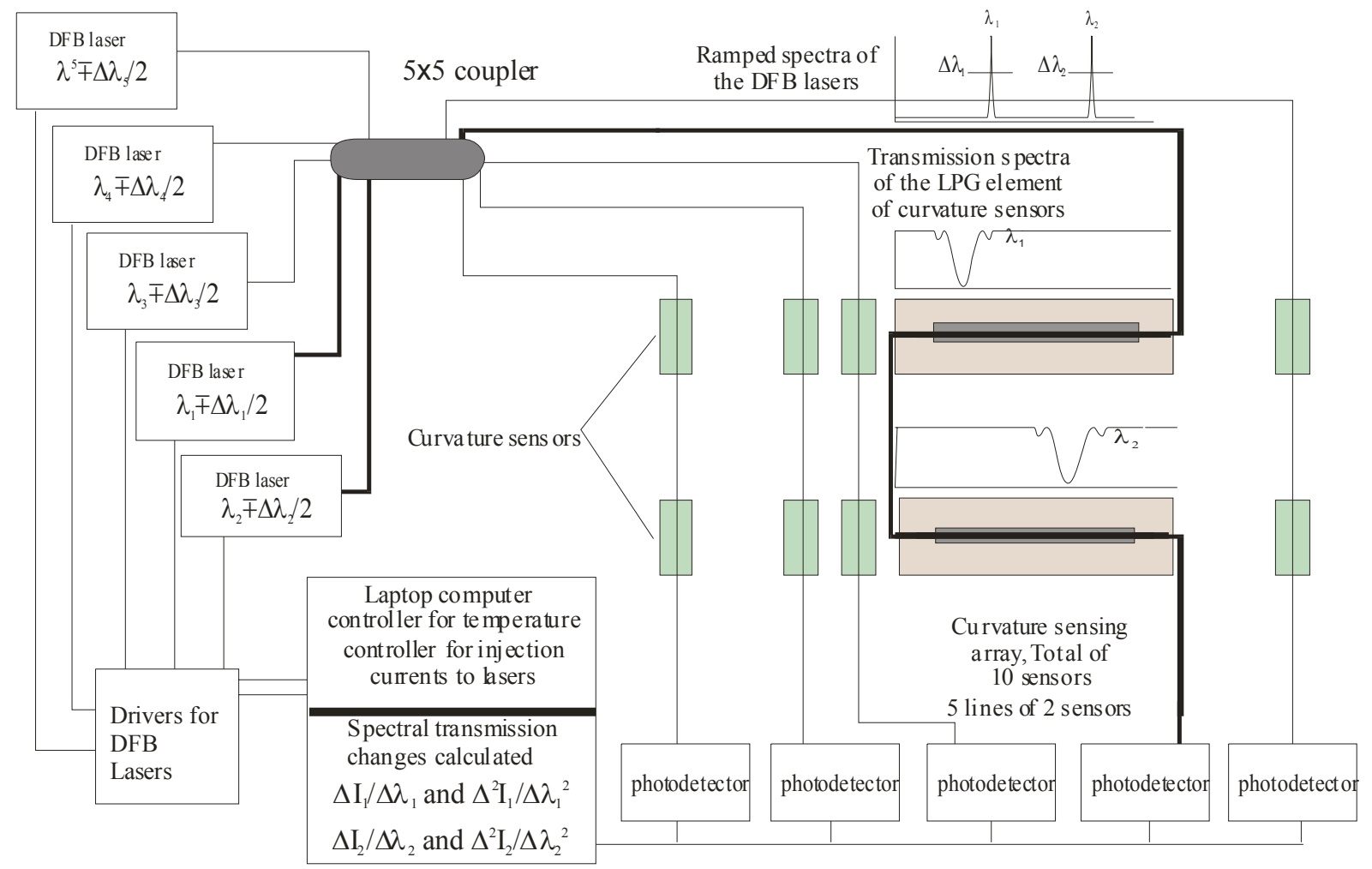

Figure 2. A schematic of interrogation/multiplexing scheme (only two lasers were used for the experimental work).

For calibration purposes, a LPG sensor was clamped between two towers; one of the clamps was mounted on a translation stage, which was moved inwards to induce a bend in the optical fibre. For this arrangement, where the LPG is mid-way between the clamps, the sensor's curvature, $R$, is given by:

$$
R=\frac{2 \cdot d}{\left(d^{2}+L^{2}\right)},
$$

where $L$ is the half distance between the edges of the two towers and $d$ is the bending displacement at the centre of the LPG.

Firstly the central wavelengths of the attenuation bands were monitored with an OSA to assess the performance of the curvature sensor in terms of curvature range and sensitivity. A typical sensor characteristic (period $=532 \times 10^{-6} \mathrm{~m}$, length $=6 \mathrm{~cm}$ ) is shown in Figure 3(a). Having obtained this information, the digital derivative spectroscopy interrogation 
technique was implemented and optimised, by adjusting the central wavelength of the DFB laser and the size of the wavelength steps. Figure 3(b) illustrates, using a parametric plot, the evolution of the amplitudes of the first and second harmonics as the curvature is varied over the range 0 to $4 \mathrm{~m}^{-1}$, while figure $3(\mathrm{c})$ is the calibration curve showing the output from the system (obtained by taking the arctangent of the ratio of the first and second harmonic amplitudes) as a function of sensor curvature.
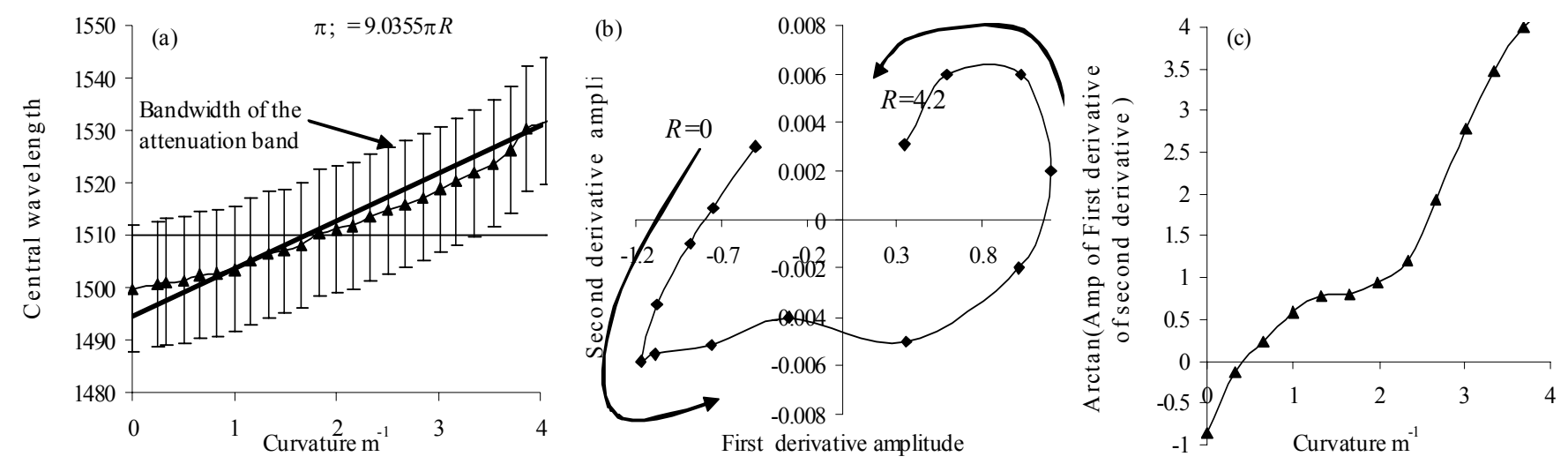

Figure 3. An example of the calibration procedure using the digital derivative spectroscopy interrogation technique. (a) The spectral response of the sensor measured using an optical spectrum analyser in order to check the usable curvature range; (b) Parametric plot of the first and second derivatives as a function of curvature; (c) Optimised output response of the sensing scheme as a function of curvature, to obtain the best linearity with maximised sensitivity.

Consideration needs to be given to the sensitivity of the sensors to other environmental parameters, the main one being temperature. Using the OSA to interrogate a curvature sensor, a spectral sensitivity of $6.87 \pm 0.11 \mathrm{~nm}$ m was found in the approximately linear response region. Modeling of the complete spectral response of the curvature sensor using a $6^{\text {th }}$ order polynomial resulted in a curvature error ( $\mathrm{rms}$ deviation from the polynomial) of $\pm 0.035 \mathrm{~m}^{-1}$. Taking into account the normal bodily temperature variation of $3^{\circ} \mathrm{C}$ leads to an overall error in curvature of $\pm 0.068 \mathrm{~m}^{-1}$. Now implementing the digital derivative spectroscopy interrogation technique introduces an error of \pm 0.062 , obtained by fitting a $6^{\text {th }}$ order polynomial to the inverse tangent of the ratio of the first and second derivatives, which translates to a similar curvature error of $\pm 0.067 \mathrm{~m}^{-1}$, and when normal bodily temperature variations are taken into account this yields a total error in curvature of $\pm 0.086 \mathrm{~m}^{-1}$.

\section{MONITORING HUMAN RESPIRATION USING A CURVATURE SENSOR ARRAY}

A sensing garment was constructed by modifying a commercially available Lycra gymnastic vest which provided a close 
contacting surface to the subject's torso. The curvature sensing array consisted of 9 sensors, the array covered the front of the torso (Rectus abdominus/Sternal Pectorallis Major region) and along the side of the torso, under the arm (Internal/External Oblique region). The array was interrogated using 2 DFB lasers, see figures 2 and 4(a). The sensors were placed in a series of pockets using Velcro ${ }^{\mathrm{TM}}$, which allowed them to be easily moved; the aim being to choose positions over the torso to enable optimal monitoring of the thoracic and abdominal movements of the subject during respiration, see figure $4(\mathrm{~b})$.

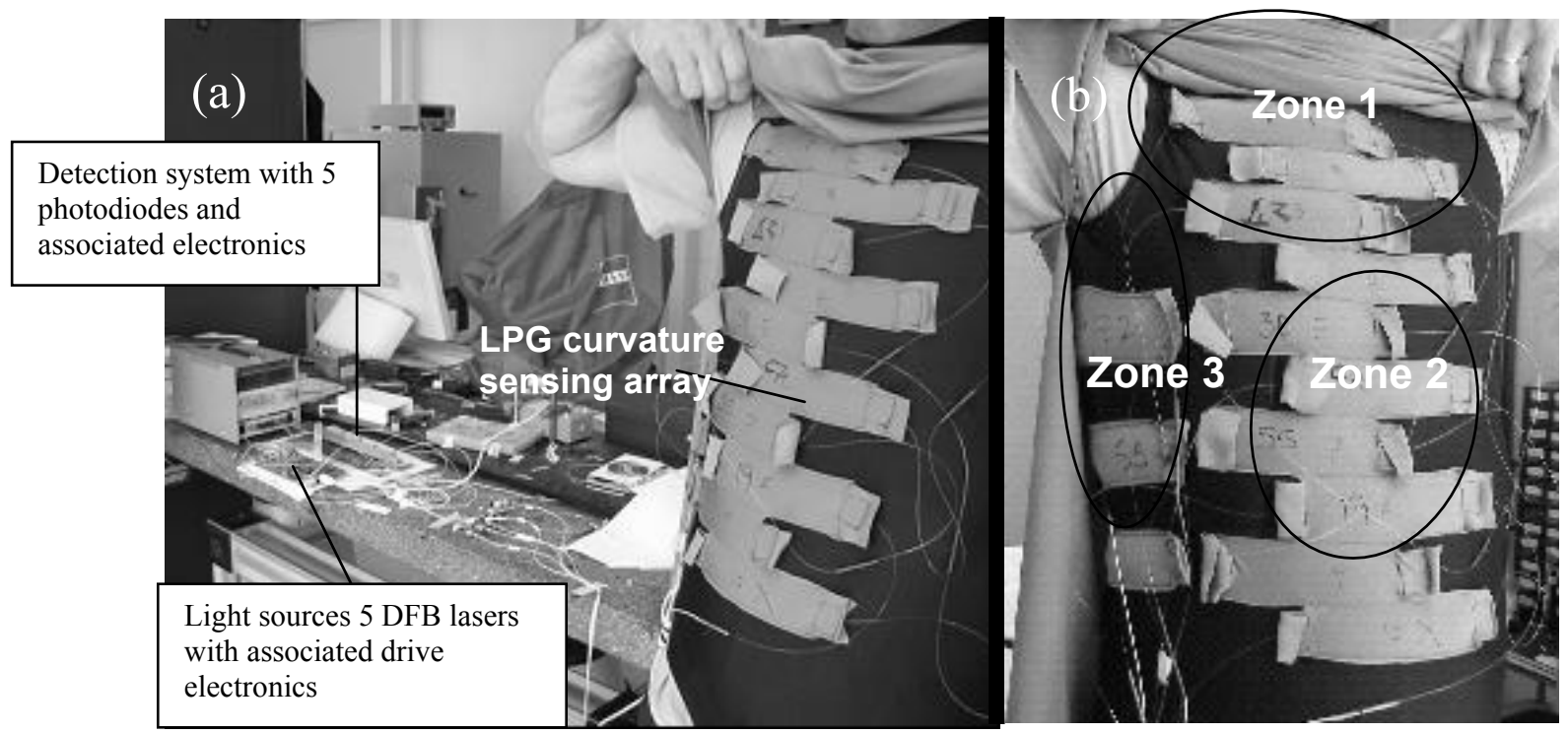

Figure 4. The curvature sensing scheme for the 9 sensors. (a) The full sensing array and the interrogator. (b) Alternative view showing the locations of the sensors on the test subject.

The sensing array was used to record curvature changes during inspiration and expiration; the flow being simultaneously recorded at the mouth of the subject using a turbine transduction spirometer system (Micro Medical, UK). The experiment involved normal rhythmic breathing and transient breathing (rapid exhalation). After the experiments were completed, linear regression was applied, enabling the curvatures obtained from the 9 sensors to predict expired volume. This process assumes that Volume $(R)=\mathrm{A} \cdot \mathrm{s}_{1}(R)+\mathrm{B} \cdot \mathrm{s}_{2}(R)+\mathrm{C} \cdot \mathrm{s}_{3}(R)+\mathrm{D} \cdot \mathrm{s}_{4}(R) \ldots+\mathrm{I} \cdot \mathrm{s}_{9}(R)$, where the constants A to I are obtained using the SPSS software package [17] and $s_{1}$ to $s_{9}$ are the responses of the individual sensors, see figures 5 . The assumption of a linear combination of the curvature sensor outputs was verified by checking that the differences between the predicted values and the observed data had a normal distribution around a mean of zero. 


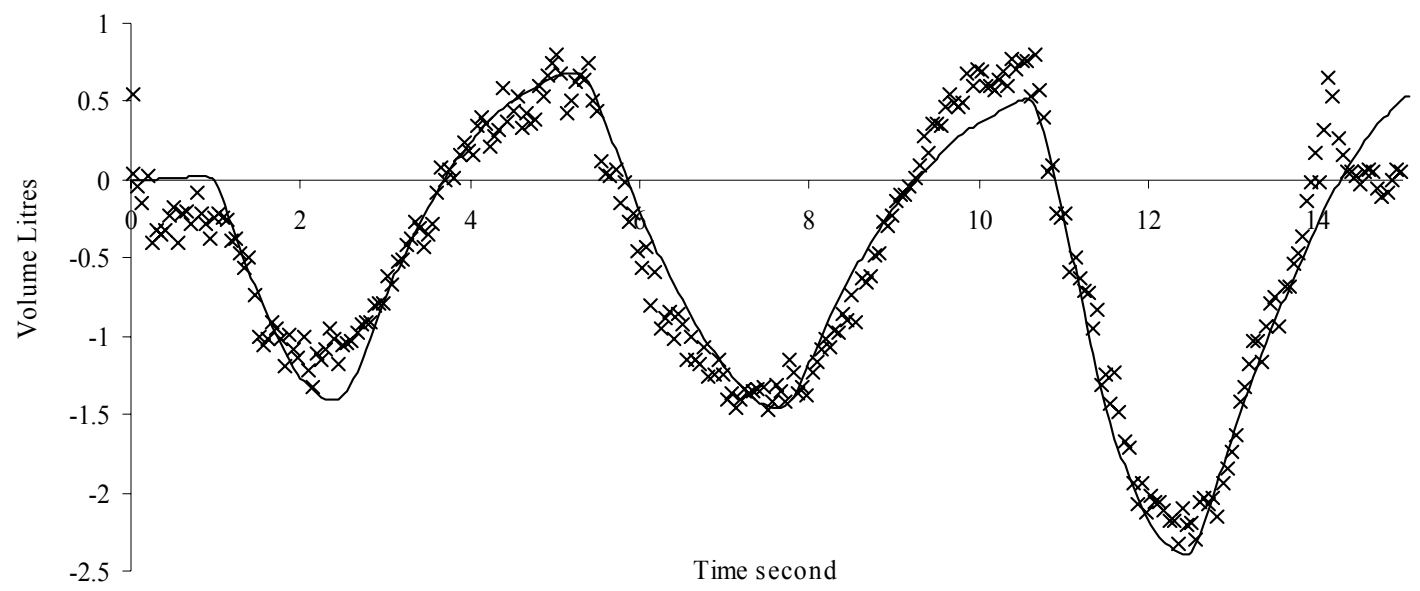

Figure 5. A typical example of normal rhythmic breathing showing the measured volume change with time from the spirometer $(-)$ and the predicted volumes for the curvature sensing scheme $(\times)$.

During testing it was found that three sensors did not produce useful outputs and did not have a significant effect on the volumetric error. Using linear regression on all the individual sensors' responses it was found that the volumetric error (one standard deviation) obtained between the curvature sensing array and the measured volume from the spirometer varied from $\sim 12 \%$ to $\sim 6 \%$ of the volume change as the number of sensors in the array varied from 4 to 9 , using this approach it was deuced that 11 to 12 sensors should be required to obtain an absolute volumetric error of $5 \%$, see figure 6a. Looking at the goodness of fit parameter shown in figure $6 \mathrm{~b}$ (the proportion of variation in the dependent variable explained by the regression model, ranging from 0 to 1), again the three under performing "failed" sensors do not make a significant contribution while inclusion of the other 6 sensors causes the goodness-of-fit to rise to 0.94 .
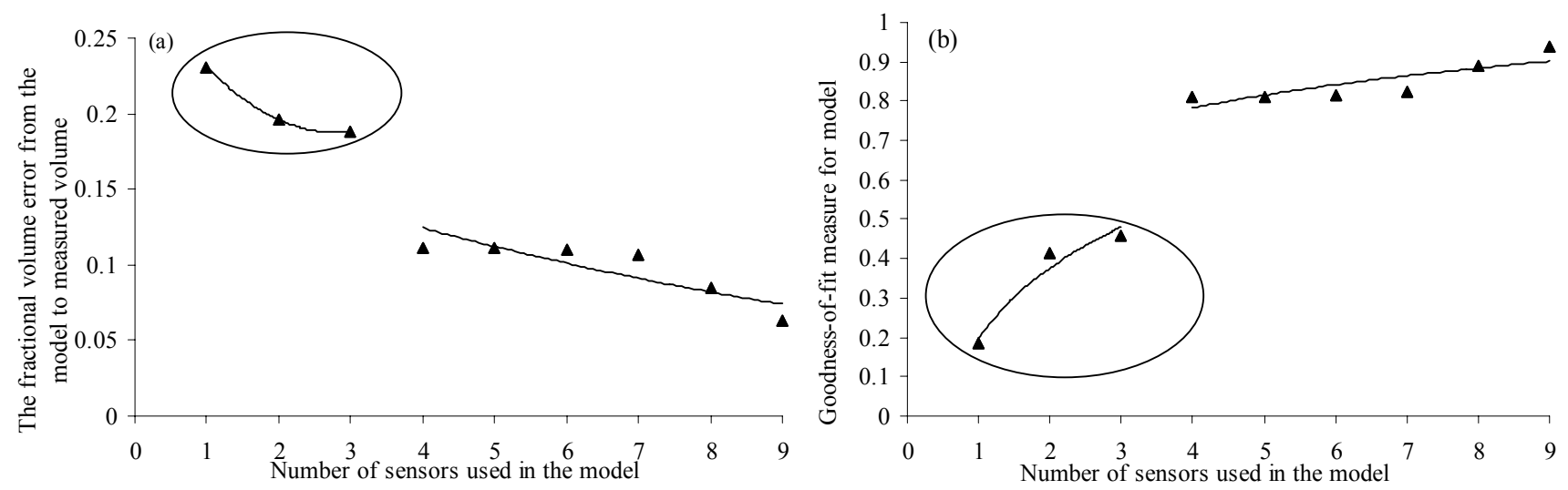

Figure 6. The performance of the curvature sensing scheme referenced to a spirometer: (a) the volumetric error from the sensing array referenced to the measured volume obtained using the spirometer as a function of the number of sensors, (b) the goodness-of-fit of the linear regression model as a function of the number of sensors. The circled data refer to the "failed" sensors.

The investigation reported above was done with a single human subject. To confirm the versatility of the 
sensing garment, it was also placed upon six other subjects with no known respiratory complaints. Subjects were chosen to cover a large variation in body shape, size and type (ectomorph, mesomorph and endomorph), see table 1. It was found that the number of sensors that did not function correctly was 0 or 1 for the majority of subjects with just one person (an ectomorph) having three failures, see table 1. This test seems to confirm that a single sensing garment can be used on a large variety of body types. It can be seen from table 1 that the sensor failures occurred in all three zones and that different sensors failed for different subjects. Failure symptoms include curvatures out of the device measurement range and large amounts of noise, perhaps caused by buckling.

Table 1 The variety of body types used with the curvature sensing garment

\begin{tabular}{|c|c|c|c|c|c|c|c|}
\hline \multirow{2}{*}{$\begin{array}{r}\text { Body } \\
\text { weight } \\
\mathrm{kg} \\
\end{array}$} & \multirow{2}{*}{$\begin{array}{l}\text { Height } \\
\mathrm{cm}\end{array}$} & \multirow{2}{*}{$\begin{array}{c}\text { Chest size } \\
\mathrm{cm}\end{array}$} & \multirow[t]{2}{*}{ Body type } & \multicolumn{3}{|c|}{$\begin{array}{c}\text { Number of } \\
\text { Array elements } \\
\text { operational }\end{array}$} & \multirow[t]{2}{*}{$\begin{array}{l}\text { Body } \\
\text { Mass } \\
\text { Index }\end{array}$} \\
\hline & & & & Zone 1 & Zone 2 & Zone 3 & \\
\hline 60 & 180 & 88.9 & ectomorph & 2 & 2 & 2 & 18.5 \\
\hline 72 & 180 & 96.5 & mesomorph & 3 & 3 & 3 & 22.5 \\
\hline 80 & 177.8 & 104.1 & mesomorph & 2 & 3 & 3 & 25 \\
\hline 82 & 157.5 & 104.1 & endomorph & 2 & 3 & 3 & 33.3 \\
\hline 98.5 & 182.9 & 108 & endomorph & 3 & 2 & 3 & 29.4 \\
\hline 105 & 195.6 & 115 & endomorph & 3 & 3 & 3 & 29.1 \\
\hline
\end{tabular}

\section{CONCLUSION}

Readings from an array of multiplexed LPG curvature sensors have been used to obtain volumetric tidal changes of the human torso which showed agreement with a spirometer used simultaneously to record the inspired and expired volume at the mouth with a volumetric error of $6 \%$ obtained with just six sensors. It was also found from statistical analysis of the sensing array data in conjunction with the measurements taken with the spirometer that 11 to 12 sensors should be required to obtain an absolute volumetric error of $5 \%$. The sensing garment was also used on a group of six human males that represented the three main different body types; ectomorph, mesomorph and endomorph corresponding to very different body mass indices; it was found that with this large variation in body shapes, the garment functioned with an average sensor failure rate of $\sim 8 \%$.

Our interrogation system based on derivative spectroscopy has the potential to be made small and portable thus enabling ambulatory or field monitoring of breathing.

\section{ACKNOWLEDGMENTS}

The authors would like to thank Fibrecore Limited, in particular Tim Hart, for supplying the PTL fibre and its specification and AEROVAC Systems Ltd for help in the development of the sensor platform, with special thanks to Matthew Fisher. The work was funded by the UK Engineering and Physical Sciences Research Council and the Wellcome Trust (grant number 074649/Z/04/Z).

\section{REFERENCES}

1 Konno, K. et al, "Measurement of the separate volume changes of the rib cage and abdomen during breathing", J. Appl. Physiol., vol.22, no.3, pp.407-422, (1967).

2 Watson $\mathrm{H}$. The technology of respiratory inductance plethysmography. In ISAM 1979. Proceedings of the third international symposium on ambulatory monitoring. ED. Scott, FD. Rafferty, EB. \& Goulding, L., PUB. London: Academic, 1980 pp537 

lung volume estimation by optical reflectance motion analysis. J. Appl. Physiol. 1996; 81(6): pp2680-2689. respiratory measurements. Jnl. Appl. Physiol. 1991; 70(5): pp2311-2321

5 Banzett, R.B., Mahan, S.T., Garner, D.M., Brughera, A. \& Loring, S.H. A simple reliable method to calibrate magnetometers and respitrace. Jnl. Appl. Physiol. 1995;79(6):pp2169-2176

6 Earthrowl-Gould T, Jones B \& Miller MR. Chest and abdominal surface motion measurement for continuous monitoring of respiratory function. Proceedings of ImechE, Part H, Engineering in medicine. (2001)

7 T. Allsop, D. Webb, I. Bennion, "A comparison and characteristics of sensing parameters of long period gratings written in three different types of fiber", Optical Fiber Technology Volume 9, Issue 4 , October 2003, Pages 210-223

8 V. Bhatia, "Applications of long-period gratings to single and multi-parameter sensing", Opt. Express, vol. 4, no. 11, pp.457-466, (1999).

9 T. Allsop, T. Earthrowl-Gould, R. Reeves, D.J. Webb, I. Bennion "The interrogation and multiplexing of Long Period Gratings curvature sensors using derivative spectroscopy technique based upon Fiber Bragg Gratings" Measurement and Science Technology vol 15, No. 1, pp. 44-48., 2004

10 H.J. Patrick, A.D. Kersey, F. Bucholtz, "Analysis of the response of long period fiber gratings to external index of refraction", J. Lightwave Technol., vol.16, no.9, pp.1606-1612, (1998).

11 H.J. Patrick, C.C. Chang, S.T. Vohra, 1998, "Long period fibre gratings for structural bend sensing", Electron. Letts., vol. 34, no. 18, pp. 1773-1775.

12 V. Bhatia, 1996, Properties and Sensing applications of long-period gratings PhDThesis, Virgina Polytechnic Institute and State University, Blackburg, Virgina.

13 M. Heiblum, J.H. Harris, "Analysis of curved optical waveguides by conformal transformation", Quant. Electron., vol.QE-11, no.2, pp. 75-83, 1975.

14 L. Zhang et al, "Design and realization of long-period grating devices in conventional and high birefringence fibers and their novel applications as fiber-optic load sensors", Selected Topics Quant. Electron., vol.5, no.5, pp. 1373-1378, (1999).

15 P. Kluczynski, 2001, "Wavelength modulation absorption spectrometry - an extensive scrutiny of the generation of signals" Spectrochimica Acta. B, Vol 56, no.8, pp1277-1354.

16 T. Allsop, R. Reeves, R. Neal, I. Bennion, 2002, "A high accuracy/sensitive refractive index sensing system based upon a long period grating Mach-Zehnder interferometer with a heterodyne detection technique", Review Sci. Instr. vol.73, no.4, pp1702-1705.

17 SPSS 12.0.1 for Windows ${ }^{\mathrm{TM}} \mathrm{http} / / / \mathrm{www}$. spss.com/ 